\title{
Charge compensation by long-period reconstruction in strongly polar lithium niobate surfaces
}

\author{
S. Sanna, ${ }^{1}$ S. Rode, ${ }^{2, *}$ R. Hölscher, ${ }^{1}$ S. Klassen, ${ }^{2}$ C. Marutschke, ${ }^{2}$ K. Kobayashi, ${ }^{3}$ H. Yamada, ${ }^{3}$ \\ W. G. Schmidt, ${ }^{1}$ and A. Kühnle, ${ }^{2,}$ \\ ${ }^{1}$ Lehrstuhl für Theoretische Physik, Universität Paderborn, 33095 Paderborn, Germany \\ ${ }^{2}$ Institut für Physikalische Chemie, Fachbereich Chemie, Johannes Gutenberg-Universität Mainz, \\ Duesbergweg 10-14, 55099 Mainz, Germany \\ ${ }^{3}$ Department of Electronic Science and Engineering, Kyoto University, Katsura, Nishikyo, Kyoto 615-8510, Japan
}

(Received 2 June 2013; revised manuscript received 5 August 2013; published 16 September 2013)

\begin{abstract}
The microscopic structure of the polar $(000 \overline{1})$ and (0001) surfaces of lithium niobate is investigated by atomic-resolution frequency modulation atomic force microscopy and first-principles calculations. It is found that the surface reconstructs at annealing temperatures sufficiently high to drive off external adsorbates. In particular a $(\sqrt{7} \times \sqrt{7}) R 19.1^{\circ}$ reconstruction is found for the $(000 \overline{1})$ surface. Density-functional theory calculations show that-apart from the $(\sqrt{7} \times \sqrt{7})$ - a series of adatom-induced surface reconstructions exist that lower the surface energy and reduce the surface charge.
\end{abstract}

DOI: 10.1103/PhysRevB.88.115422

PACS number(s): 68.35.B-, 68.08.-p, 68.37.Ps, 68.43.-h

Ferroelectric oxides-and lithium niobate $\left(\mathrm{LiNbO}_{3}, \mathrm{LN}\right)$ in particular-have become extremely important in numerous areas of broad technological significance, being the main component of modulators, wavelength filters, secondharmonic generators, and nonvolatile memories. ${ }^{1}$ While these applications exploit the peculiar ferroelectric, piezoelectric, photorefractive, and electro-optical properties of the bulk, the strong electric fields and charges at the surfaces of ferroelectric materials have recently attracted the attention of scientists and engineers. For example, it is possible to grow group III nitrides with spatially varied, absolute polarity control on LN substrates. ${ }^{2}$ The dipole orientation can be switched at the nanoscale, bearing a great potential for domain-specific surface chemistry as a route towards the fabrication of nanoscale devices. ${ }^{3} \mathrm{LN}$ surface charges were found to enable artificial photosynthesis ${ }^{4}$ and to drive photocatalytic dye decolorization. ${ }^{5}$ The integration of ferroelectric thin films within liquid environments is investigated in the context of laboratory-on-chip device designs, e.g., for localizing, sensing or activating charged biomolecules. ${ }^{6}$ High surface electric fields due to pyroelectricity were found to efficiently pole electro-optic polymers ${ }^{7}$ and to lead to the reversible fragmentation and self-assembling of nematic liquid crystals. ${ }^{8}$

Despite many exciting applications, our actual knowledge of the LN surface atomic structure and the associated surface electric field and surface charge is remarkably limited. $\mathrm{LiNbO}_{3}$ can be thought of as a stapling of $\mathrm{Nb}_{-} \mathrm{O}_{3}-\mathrm{Li}$ trilayers along the [0001] direction (Fig. 1). This order gives rise to a spontaneous electric dipole moment and strongly polar (0001) and (0001) surfaces, commonly referred to as negative and positive $Z$ cut, respectively. Using electron diffraction, Yun et $a l .{ }^{9}$ found no indication for reconstructions on $\mathrm{LN}(0001)$ surfaces at ambient conditions. From ion scattering spectroscopy it was concluded that both $Z$-cut surfaces are oxygen terminated. ${ }^{9}$ On the other hand, coaxial impact-collision ion scattering suggested a $\mathrm{Nb}$ surface termination. ${ }^{10,11}$ The experimental investigations are hindered by the extremely challenging preparation and analysis conditions of strongly polar surfaces of insulating materials: Charging effects preclude the application of electron tunneling or diffraction techniques and unscreened surface charges hinder atomic force microscopy. Nonetheless, reconstructions in surfaces commonly referred to as weakly polar surfaces such as the $\mathrm{SrTiO}_{3}(001)$ and $\mathrm{BaTiO}_{3}(001)$, have been recently demonstrated by STM $^{12,13}$ and transmission electron microscopy ${ }^{14}$ techniques. Moreover, the structural and physical properties of polar surfaces are strongly temperature dependent. ${ }^{15}$ Only recently, atomic resolution images of the $\mathrm{LN}(000 \overline{1})$ surface have been obtained by frequency modulation atomic force microscopy (FM-AFM) operated at the solid-water interface. ${ }^{16}$ However, these experiments were performed on samples after annealing at $1270 \mathrm{~K}$, and no surface reconstruction was observed. Similarly, the calculated surface phase diagrams of LN Z-cut surfaces-while consistent with the AFM data ${ }^{16}$-have been restricted to unreconstructed geometries so far. ${ }^{17-19}$

In the present work we combine AFM measurements with density-functional theory (DFT) calculations and (i) demonstrate that adsorbate-free LN Z-cut surfaces reconstruct, (ii) explain the surface reconstruction microscopically in terms of favorable adatom structures, and (iii) show that these reconstructions lower the surface polarization charge.

Commercially available single-crystal $Z$-cut wafers (9900042-01, Crystal Technology, Inc., USA) were prepared as described previously. ${ }^{16}$ Annealing was performed for 5 hours in a muffle furnace from Nabertherm $\mathrm{GmbH}$ (Lilienthal, Germany) at the indicated temperatures. The temperature setting of the furnace was tested with an additional thermocouple, confirming the temperature settings with an error of approximately $\pm 5 \mathrm{~K}$. The atomic force microscope (MultiMode V from Bruker Nano Surfaces Division, USA) was operated in tapping mode for micrometerscale imaging. Atomic-resolution images were obtained in water using FM-AFM ${ }^{20}$ following the procedure described in Ref. 16. 


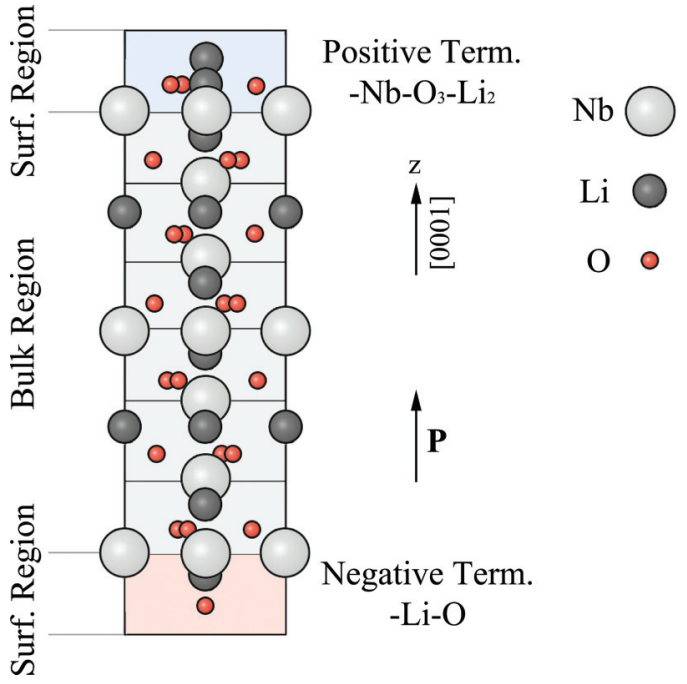

FIG. 1. (Color online) Schematic representation of $\mathrm{LiNbO}_{3}$ along the [0001] direction indicating (charge neutral) bulk and surface regions.

A series of micrometer-scale AFM images of the (0001) and (0001) surfaces taken in air is given in Fig. 2. These images illustrate the dependence of the surface morphology on the annealing procedure. In Figs. 2(a) and 2(e), images are shown that are taken directly after ultrasonification without further annealing. The surfaces exhibit a large number of adsorbed species and a high surface roughness. In particular, no features that can be associated with surface step edges can be identified. The situation changes when annealing the samples after ultrasonification. Different samples were prepared annealed at different temperatures with $100 \mathrm{~K}$ temperature steps. No drastic change is observed for an annealing temperature of $870 \mathrm{~K}$, as shown in Figs. 2(b) and 2(f). A significant change in morphology is observed after annealing to $1070 \mathrm{~K}$ (referred to as moderate annealing step), as shown in Figs. 2(c) and $2(\mathrm{~g})$, revealing a highly structured surface with atomically flat hexagonal terraces. No adsorbates are found on these terraces. The straight lines observed in these images can be identified as step edges, formed by multiples of a monatomic step. Yet another change is observed after annealing to 1270 K. Representative images obtained after this hightemperature annealing step-corresponding to the preparation conditions in Ref. 16-are presented in Figs. 2(d) and 2(h). Again, atomically flat terraces are observed. The hexagonal terraces have vanished, however, and relatively straight steps appeared.

To elucidate the details of this morphology change, we performed high-resolution FM-AFM at the solid-water interface on samples that were prepared as shown in Figs. 2(c) and 2(d). The samples-we are focusing on $\operatorname{LN}(000 \overline{1})$ in the following - were allowed to equilibrate to room temperature after annealing. An image taken after high-temperature annealing to $1270 \mathrm{~K}$ (cf. Ref. 16) is shown in Fig. 3(a). A hexagonal surface structure is observable in real space and corroborated by the Fourier transform in Fig. 3(c). It corresponds to a real-space unit cell with a lattice constant of $0.515 \pm 0.020 \mathrm{~nm}$.
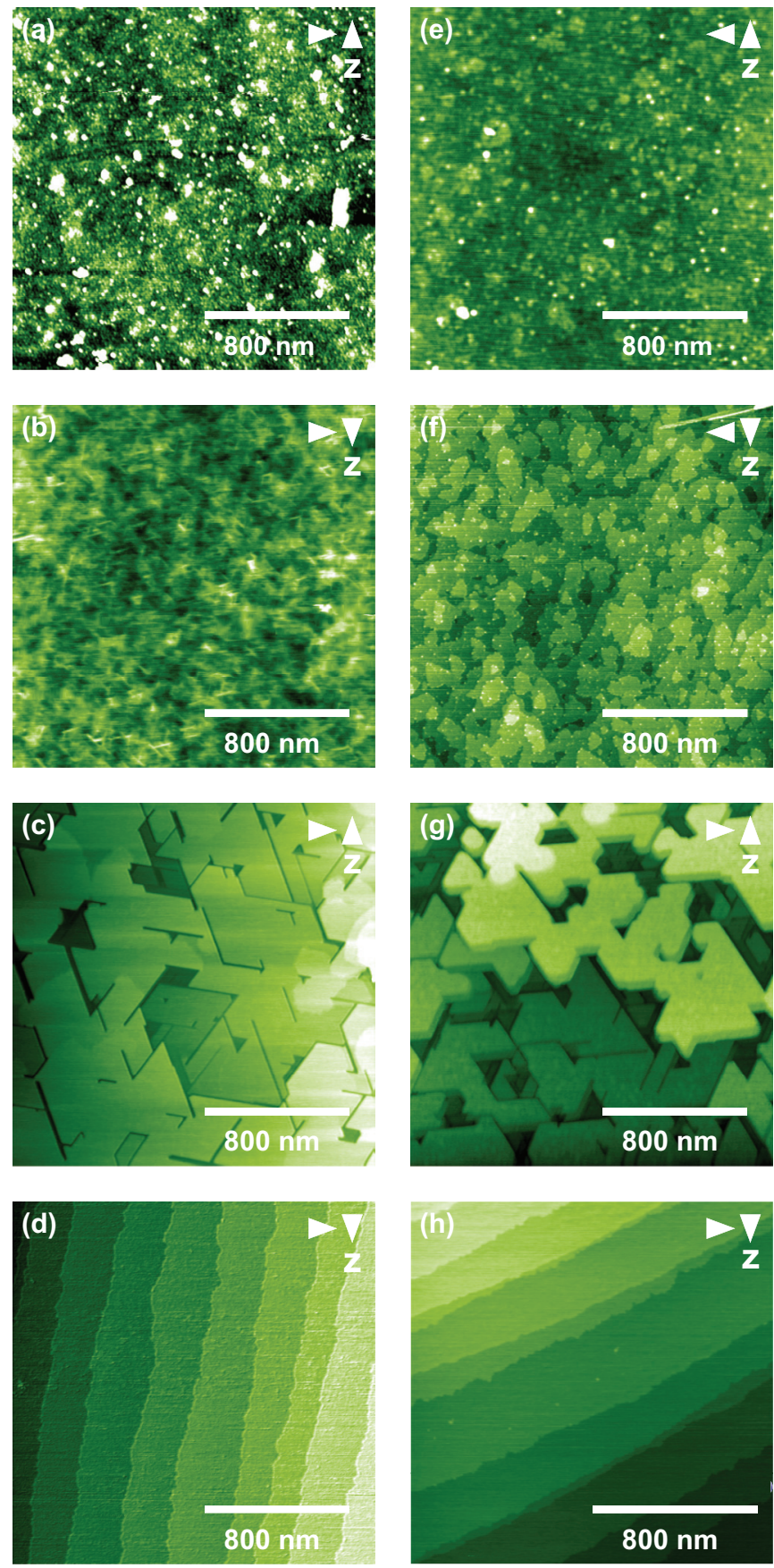

FIG. 2. (Color online) Tapping mode AFM images of the (000 $\overline{1})$ (left-hand side) and (0001) (right-hand side) surfaces taken in air after cleaning in an ultrasound bath without further annealing [(a) and (e)], after subsequent annealing at $870 \mathrm{~K}$ [(b) and (f)], at $1070 \mathrm{~K}$ [(c) and $(\mathrm{g})]$, and at $1270 \mathrm{~K}[(\mathrm{~d})$ and $(\mathrm{h})]$.

This finding is consistent with a bulk-truncated structure of the $(000 \overline{1})$ surface.

Interestingly, the moderate annealing step to $1070 \mathrm{~K}$ leads to a different atomic structure, as shown in Figs. 3(b) and 3(d). Again, a hexagonal structure is observed. However, as confirmed by Fourier analysis, the lattice constant is considerably larger and amounts to $1.362 \pm 0.02 \mathrm{~nm}$. This corresponds to a $(\sqrt{7} \times \sqrt{7}) R 9.1^{\circ}$ surface reconstruction, as shown in Fig. 3(e). 

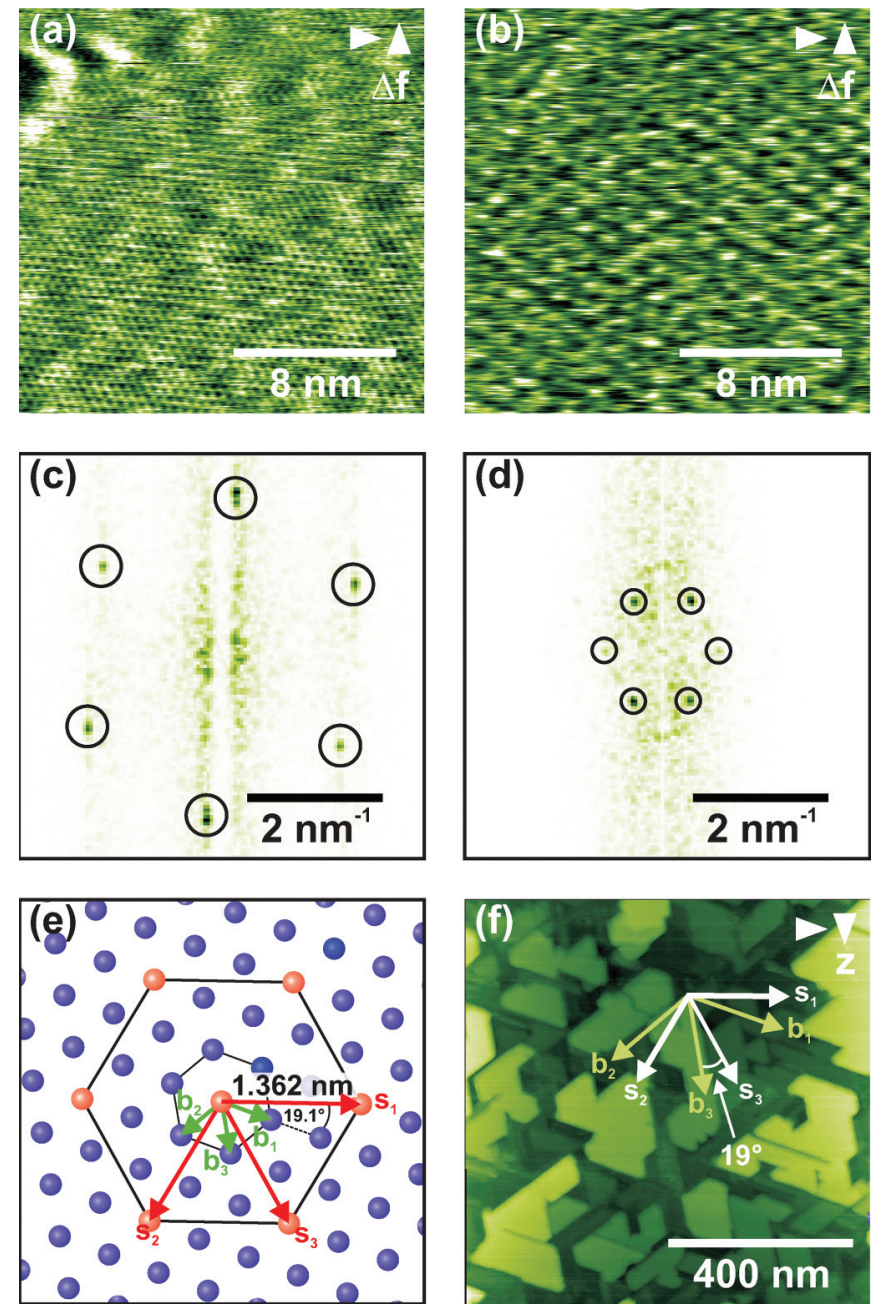

FIG. 3. (Color online) Atomically resolved NC-AFM images of the $\mathrm{LN}(000 \overline{1})$ after (a) high-temperature annealing and (b) moderate annealing. Corresponding Fourier transformed images (c) and (d), $(\sqrt{7} \times \sqrt{7}) R 19.1^{\circ}$ reconstruction model (e), and relative orientation of the bulk unit cell vectors $\left(\mathbf{b}_{1}, \mathbf{b}_{2}, \mathbf{b}_{3}\right)$ to the surface step edges, showing a $19.1^{\circ}$ rotation (f).

The orientation of the surface reconstruction (given by $\mathbf{s}_{1}$, $\mathbf{s}_{2}$, and $\mathbf{s}_{3}$ ) is compared with the direction of the step edges in Fig. 3(f). The two directions are rotated with respect to each other by $19.1^{\circ}$, which is exactly what we expect for the relative orientation of the reconstruction vectors and the bulk vectors $\left(\mathbf{b}_{1}, \mathbf{b}_{2}\right.$, and $\left.\mathbf{b}_{3}\right)$. This indicates that the step edges in the reconstructed phase are aligned along the bulk lattice directions.

To assign a microscopic structure to the AFM findings discussed above we perform density-functional calculations. Thereby the VASP implementation ${ }^{21}$ within the generalizedgradient approximation $^{22}$ is used. The technical details of the calculations are equivalent to Ref. 18 . In contrast to previous LN surface calculations, ${ }^{17-19}$ the possibility of surface reconstructions was taken into account, probing $(\sqrt{3} \times \sqrt{3})$ and $(\sqrt{7} \times \sqrt{7})$ structures.
Levchenko and Rappe ${ }^{17}$ have used the formal oxidation states $q_{N b}=5 e, q_{O}=-2 e$, and $q_{L i}=e$ in conjunction with Berry phase theory ${ }^{23,24}$ to estimate the surface charge for the clean, unreconstructed $\mathrm{LN} Z$-cut surfaces. The thermodynamically favorable $-\mathrm{Li}-\mathrm{O} /-\mathrm{Nb}-\mathrm{O}_{3}-\mathrm{Li}_{2}$ terminated $\mathrm{LN}(000 \overline{1}) /(0001)$ surfaces were found to have net surface charges of $+e / 4$ and $-e / 4$, respectively. In the present study we focused on stoichiometries that-within the model of formal oxidation states-further reduce this surface charge. About 200 configurations modeling about 40 stoichiometries were probed for each of the $(\sqrt{7} \times \sqrt{7})$ and the $(\sqrt{3} \times \sqrt{3})$ reconstructions. The resulting surface phase diagrams in dependence on the oxygen and lithium chemical potentials are shown in Fig. 4. The present results closely resemble earlier findings ${ }^{17,18}$ for Li-poor environments. However, reconstructed surfaces are predicted for a wide range of the thermodynamically accessible conditions $^{25}$ that are indicated by the trapezoids in Fig. 4. In particular, various stable $(\sqrt{7} \times \sqrt{7})$ reconstructions are induced by $\mathrm{Li}$ and $\mathrm{O}$ adatoms. While we cannot exclude that further reconstructions are even more favorable, our results clearly show that unreconstructed LN surfaces are stable only in a rather limited range of surface preparation conditions. The pressure and temperature dependence of the chemical potentials ${ }^{26}$ assigns the encircled areas in the phase diagrams to moderate annealing conditions. Such conditions thus favor $(\sqrt{3} \times \sqrt{3}) R 30^{\circ}$ and $(\sqrt{7} \times \sqrt{7}) R 19.1^{\circ}$ reconstructions induced by single $\mathrm{O}$ and $\mathrm{Li}, \mathrm{O}$ adatoms, respectively, for the negative $Z$ cut. Corresponding conditions for the positive $Z$ cut favor various Li-induced $(\sqrt{7} \times \sqrt{7})$ structures. The thermodynamically most dominant $(000 \overline{1})(\sqrt{7} \times$ $\sqrt{7})+\mathrm{LiO}$ and $(0001)(\sqrt{7} \times \sqrt{7})+\mathrm{Li}_{2}$ surfaces are shown in Figs. 5(a) and 5(b), respectively. Assuming a lithium partial pressure lower than $10^{-6} \mathrm{~Pa}, a b$ initio thermodynamics in conjunction with the ideal gas approximation ${ }^{26}$ predicts the $\sqrt{7} \times \sqrt{7}$ reconstructions to be stable roughly from 750 to $1150 \mathrm{~K}$, in reasonable agreement with the AFM data.

What is driving the formation of surface reconstructions on LN? The surface polarization charge plays a decisive role for the stability of ferroelectric surfaces. It is expected to be largely compensated by a change of the surface stoichiometry as well as adsorbates. ${ }^{12,27}$ In fact, the charges measured for LN scatter considerably, ${ }^{28}$ but are far below the $0.7 \mathrm{C} / \mathrm{m}^{2}$ expected from the bulk polarization. Recently, a value of $\sigma=140 \mu \mathrm{C} / \mathrm{m}^{2}$ for congruent, undoped $z$-faced $\mathrm{LiNbO}_{3}$ crystals under ambient conditions has been determined. ${ }^{29}$ In the absence of external adsorbates, internal mechanisms are required to reduce the surface polarization charge. ${ }^{30,31}$ In order to quantify the role of surface reconstructions in this context, we perform a Bader analysis $^{32,33}$ and find that the surface charge is reduced by all stable reconstructions. The $(\sqrt{7} \times \sqrt{7})+\mathrm{LiO}$ and the $(\sqrt{3} \times \sqrt{3})+O$ terminations at the negative $Z$ cut reduce the surface charge by $9 \%$ and $11 \%$ with respect to the unreconstructed surface, respectively. At the positive $Z$ cut, a surface charge reduction by $17 \%, 15 \%, 12 \%$, and $3 \%$ occurs for the $(\sqrt{7} \times \sqrt{7})+\mathrm{Li}_{3},(\sqrt{3} \times \sqrt{3})+\mathrm{Li},(\sqrt{7} \times$ $\sqrt{7})+\mathrm{Li}_{3}$, and $(\sqrt{7} \times \sqrt{7})+\mathrm{Li}$ structures, respectively. We find the calculated surface charge reduction to be roughly 

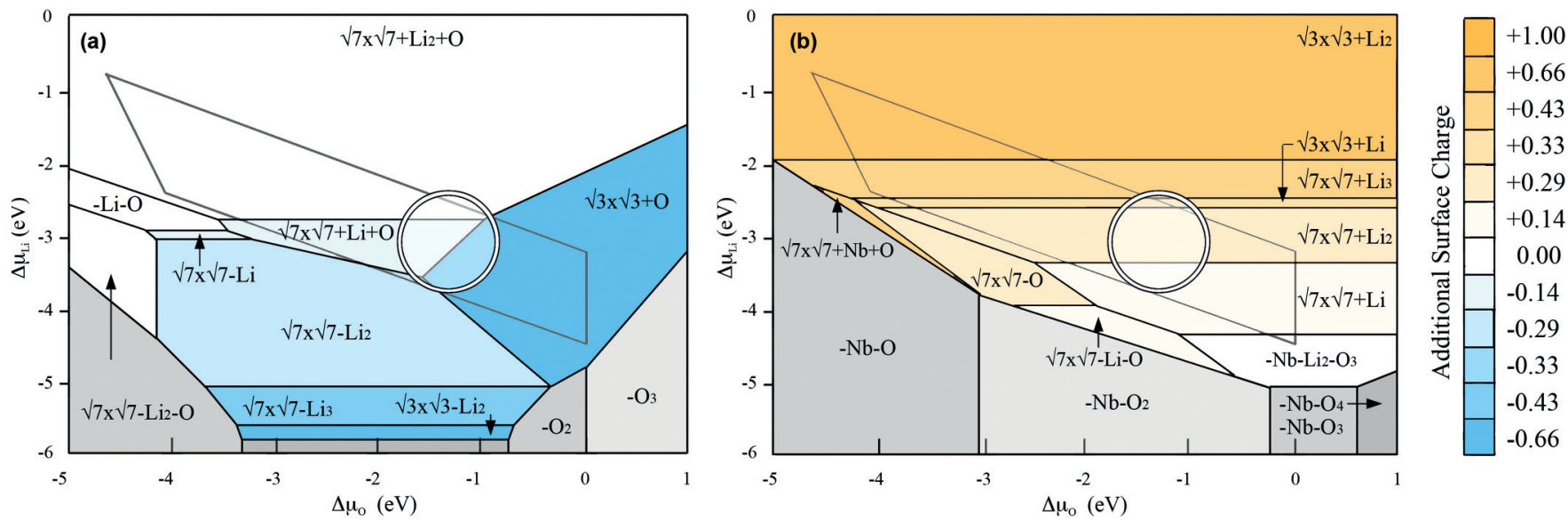

FIG. 4. (Color online) Calculated phase diagrams of the $\mathrm{LN}(000 \overline{1}) /(0001)$ surface in dependence on the $\mathrm{O}$ and $\mathrm{Li}$ chemical potentials $\mu$ are shown in (a) and (b). The range of $\mu_{O}$ and $\mu_{L i}$ accessible in thermodynamic equilibrium is within the trapezoids. The experimental conditions that result in the surfaces shown in Figs. 2(b) and 2(e) are indicated by the encircled areas. The calculated charge compensation (with respect to the $-\mathrm{Li}-\mathrm{O} /-\mathrm{Nb}-\mathrm{O}_{3}-\mathrm{Li}_{2}$ terminated $\mathrm{LN}(000 \overline{1}) /(0001)(1 \times 1)$ surface $)$ is color coded. Unreconstructed structures previously identified in Refs. $17-19$ with calculations restricted to the $1 \times 1$ periodicity are gray.

proportional to the nominal charge of the respective adatoms. While these calculations show that LN surface reconstructions are, indeed, reducing the surface polarization charge, we find the charge compensation by external mechanisms such as charged adatoms and foreign adsorbates to be more efficient. For example, the adsorption of $\mathrm{Li}^{+}$or $\mathrm{H}$ on the positive $(\sqrt{3} \times \sqrt{3})$ surface reduces the calculated surface charge by about $41 \%$ or $31 \%$.

The combination of AFM measurements and DFT calculations thus provides a comprehensive picture of the temperature-dependent stabilization mechanisms of polar LN $Z$ cuts. At ambient conditions and for low annealing temperatures the surface charge is mainly compensated by foreign adsorbates, explaining why no surface reconstruction
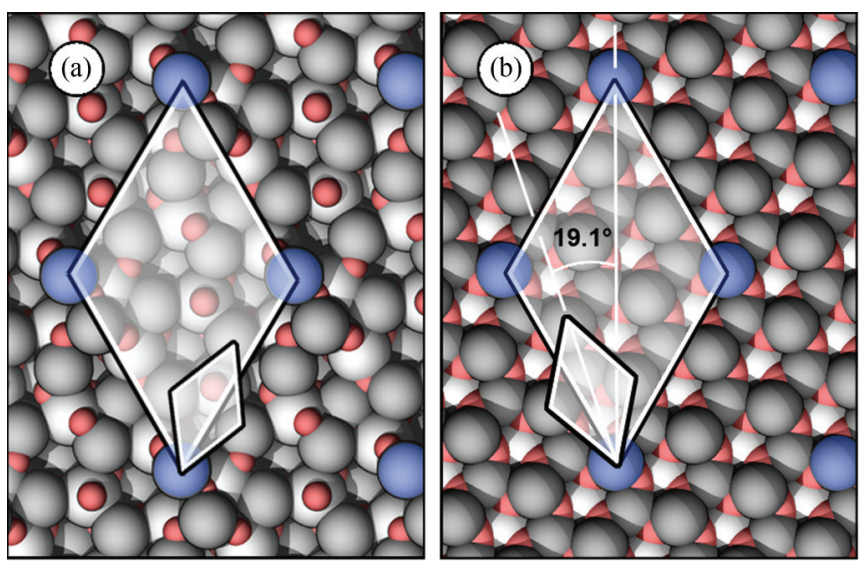

FIG. 5. (Color online) Calculated $(000 \overline{1})(\sqrt{7} \times \sqrt{7})+\mathrm{LiO}$ (a) and $(0001)(\sqrt{7} \times \sqrt{7})+\mathrm{Li}_{2}$ (b) surfaces predicted for moderate annealing. The surface reconstructions as well as the primitive surface unit cells are indicated. Color coding as in Fig. 1, with the uppermost $\mathrm{Li}$ adatoms shown in blue. was observed. At elevated temperatures, however, the adsorbates start to desorb (see also, e.g., Ref. 34). Above about $1000 \mathrm{~K}$ the surface is completely adsorbate free. This requires the formation of surface reconstructions in order to avoid polar instabilities due to uncompensated surface charges. The $\operatorname{LN}(000 \overline{1})(\sqrt{7} \times \sqrt{7}) R 19.1^{\circ}$ surface observed here upon moderate annealing is one example in this respect. Upon high-temperature annealing, finally, the surface recovers the bulk periodicity. This to be expected for entropy reasons as well as due to the reduced polarization as the Curie temperature is approached, reducing the driving force for the formation of surface reconstructions. However, one should keep in mind that due to the strong ferro-, pyro-, and piezoelectric nature of the crystal, already small variations in the atmosphere composition, temperature, and pressure will result in an underor overcompensation of the polarization charge, ${ }^{29}$ so that the net surface charge might even change sign or oscillate around the neutral state.

Summarizing, we have investigated the temperaturedependent evolution of the LN Z-cut morphology by means of frequency-modulated AFM and presented evidence for the formation of surface reconstructions that form upon annealing the sample to drive off adsorbates from the ambient. Ab initio calculations confirm the stability of LN surface reconstructions for a wide range of preparation conditions and explain them in terms of adatom structures that contribute to the internal charge compensation of the strongly polar surfaces. The present work demonstrates microscopically the various charge compensation mechanisms that prevent the electrostatic instability of polar ferroelectric surfaces.

This work has been supported by the DFG and BASF SE. The calculations were performed using grants of computer time from the Paderborn $\mathrm{PC}^{2}$ and the HLRS Stuttgart. 
*Now at SmarAct GmbH.

${ }^{\dagger}$ kuehnle@uni-mainz.de

${ }^{1} \mathrm{Y}$. Xu, Ferroelectric Materials and their Applications (Elsevier, Amsterdam, 1991).

${ }^{2}$ G. Namkoong, K.-K. Lee, S. M. Madison, W. Henderson, S. E. Ralph, and W. A. Doolittle, Appl. Phys. Lett. 87, 171107 (2005).

${ }^{3}$ D. Li, M. H. Zhao, J. Garra, A. M. Kolpak, A. M. Rappe, D. A. Bonnell, and J. M. Vohs, Nat. Mater. 7, 473 (2008).

${ }^{4}$ M. Stock and S. Dunn, IEEE Trans. Ultrason. Ferroelectr. Freq. Control 58, 1988 (2011).

${ }^{5}$ M. Stock and S. Dunn, J. Phys. Chem. C 116, 20854 (2012).

${ }^{6}$ R. Ferris, B. Yellen, and S. Zauscher, Small 8, 28 (2011).

${ }^{7}$ S. Huang, J. Luo, H.-L. Yip, A. Ayazi, X.-H. Zhou, M. Gould, A. Chen, T. Baehr-Jones, M. Hochberg, and A. K. Y. Jen, Adv. Mater. 24, OP42 (2011).

${ }^{8}$ F. Merola, S. Grilli, S. Coppola, V. Vespini, S. De Nicola, P. Maddalena, C. Carfagna, and P. Ferraro, Adv. Funct. Mater. 22, 3267 (2012).

${ }^{9}$ Y. Yun, M. Li, D. Liao, L. Kampschulte, and E. Altman, Surf. Sci. 601, 4636 (2007).

${ }^{10}$ A. Saito, H. Matsumoto, S. Ohnisi, M. Akai-Kasaya, Y. Kuwahara, and M. Aono, Jpn. J. Appl. Phys. 43, 2057 (2004).

${ }^{11}$ K. Tabata, T. Choso, A. Murakami, and E. Suzuki, Surf. Sci. 402, 487 (1998).

${ }^{12}$ John Mark P. Martirez, E. H. Morales, W. A. Saidi, D. A. Bonnell, and A. M. Rappe, Phys. Rev. Lett. 109, 256802 (2012).

${ }^{13}$ M. R. Castell, Surf. Sci. 505, 1 (2002).

${ }^{14}$ G.-Z. Zhu, G. Radtke, and G. A. Botton, Nature (London) 490, 384 (2012).

${ }^{15}$ B. C. Russell and M. R. Castell, Phys. Rev. B 75, 155433 (2007).

${ }^{16}$ S. Rode, R. Hölscher, S. Sanna, S. Klassen, K. Kobayashi, H. Yamada, W. G. Schmidt, and A. Kühnle, Phys. Rev. B 86, 075468 (2012).

${ }^{17}$ S. V. Levchenko and A. M. Rappe, Phys. Rev. Lett. 100, 256101 (2008).
${ }^{18}$ S. Sanna and W. G. Schmidt, Phys. Rev. B 81, 214116 (2010).

${ }^{19}$ S. Sanna, A. V. Gavrilenko, and W. G. Schmidt, Phys. Status Solidi C 7, 145 (2010).

${ }^{20}$ S. Rode, R. Stark, J. Lübbe, L. Tröger, J. Schütte, K. Umeda, K. Kobayashi, H. Yamada, and A. Kühnle, Rev. Sci. Instrum. 82, 073703 (2011).

${ }^{21}$ G. Kresse and J. Furthmüller, Comput. Mater. Sci. 6, 15 (1996).

${ }^{22}$ J. P. Perdew, J. A. Chevary, S. H. Vosko, K. A. Jackson, M. R. Pederson, D. J. Singh, and C. Fiolhais, Phys. Rev. B 46, 6671 (1992).

${ }^{23}$ D. Vanderbilt and R. D. King-Smith, Phys. Rev. B 48, 4442 (1993).

${ }^{24}$ R. Resta and D. Vanderbilt, in Physics of Ferroelectrics: A Modern Perspective (Springer, Berlin, 2007).

${ }^{25}$ H. Xu, D. Lee, J. He, S. B. Sinnott, V. Gopalan, V. Dierolf, and S. R. Phillpot, Phys. Rev. B 78, 174103 (2008).

${ }^{26}$ C. G. Van de Walle and J. Neugebauer, Phys. Rev. Lett. 88, 066103 (2002).

${ }^{27}$ J. Goniakowski, F. Finocchi, and C. Noguera, Rep. Prog. Phys. 71, 016501 (2008)

${ }^{28}$ T. Jungk, Á. Hoffmann, and E. Soergel, Appl. Phys. Lett. 89, 042901 (2006).

${ }^{29}$ F. Johann and E. Soergel, Appl. Phys. Lett. 95, 232906 (2009).

${ }^{30}$ O. Dulub, U. Diebold, and G. Kresse, Phys. Rev. Lett. 90, 016102 (2003).

${ }^{31}$ M. Kunat, S. G. Girol, T. Becker, U. Burghaus, and C. Wöll, Phys. Rev. B 66, 081402 (2002).

${ }^{32}$ R. F. W. Bader, Atoms in Molecules - A Quantum Theory (Oxford University Press, Oxford, 1990).

${ }^{33}$ Test calculations for the unreconstructed surface show that the Bader analysis results agree with the outcome of Berry phase calculations of the total slab polarization (Refs. 23 and 24). The polarization of the truncated bulk is calculated to be $0.69 \mathrm{C} / \mathrm{m}^{2}$, which is in excellent agreement with the measured LN bulk polarization.

${ }^{34}$ J. Garra, J. M. Vohs, and D. A. Bonnell, Surf. Sci. 603, 1106 (2009). 